\title{
Role of piezoelectricity in dielectric response of Rochelle salt type crystals
}

\author{
R.R.Levitskii ${ }^{1}$, I.R.Zachek ${ }^{2}$, T.M.Verkholyak ${ }^{1}$, A.P.Moina ${ }^{1}$ \\ 1 Institute for Condensed Matter Physics \\ of the National Academy of Sciences of Ukraine, \\ 1 Svientsitskii Str., 79011 Lviv, Ukraine \\ 2 National University "Lvivs'ka Politekhnika", \\ 12 Bandera Str., 79013 Lviv, Ukraine
}

Received December 9, 2002

Comparing the spontaneous polarizations, static and dynamic dielectric properties of piezoelectric Rochelle salt and non-piezoelectric $\mathrm{RbHSO}_{4}$ we explore the role played by piezoelectric coupling in forming the dielectric response of the crystals of this type. The calculations for crystals of both types are performed within the Mitsui model, modified for the case of Rochelle salt by including the terms related to piezoelectric coupling with spontaneous strain $\varepsilon_{4}$. It is shown that such a modification improves the agreement between theory and experiment for spontaneous polarization and yields a correct temperature behavior of relaxation times and dynamic dielectric permittivity of Rochelle salt in the vicinity of the transition points.

Key words: Rochelle salt, $\mathrm{RbHSO}_{4}$, piezoeffect, relaxation dynamics, polarization, clamping

PACS: $77.22 . \mathrm{Gm}, 77.65 .-j$, 77.80.Bh

\section{Introduction}

Both piezoelectric Rochelle salt and non-piezoelectric $\mathrm{RbHSO}_{4}$ belong to the same class of ferroelectrics, usually described by a two-sublattice model of dipoles moving in asymmetric double potentials (Mitsui model [1]). In Rochelle salt it has not been established which atoms play the role of the ordering units, whereas in $\mathrm{RbHSO}_{4}$ the dipoles are usually attributed to sulphate groups. The model, depending on the values of its parameters, can describe the peculiar to Rochelle salt two second order phase transitions with the ferroelectric phase between them, as well as the observed in $\mathrm{RbHSO}_{4}$ single second order phase transition into the ferroelectric phase.

Calculations performed for Rochelle salt within the conventional Mitsui model usually face two important problems: i) impossibility to simultaneously fit the spon- 
taneous polarization and the static permittivity (too small polarization is obtained if the permittivity is fitted, or too large Curie constant is observed when polarization is correct) [2,3], and ii) incorrect temperature dependence of relaxation times and dynamic permittivity at the Curie points $[2,4-8]$ : One of the inverse relaxation times as well as a contribution of the ordering subsystem to the permittivity vanish at the Curie points, whereas experiments [9] indicate that both should be finite.

Both problems are not encountered in a non-piezoelectric $\mathrm{RbHSO}_{4}$ crystal. Here, the inverse relaxation times and dynamic permittivity (theoretical and experimental) actually vanish at the Curie point $[2,10-12]$ and a good description of spontaneous polarization and static dielectric susceptibility can be obtained.

These facts plainly indicate that the piezoelectric effects play an important role in forming the dielectric response of Rochelle salt crystals. An impossibility to obtain a correct temperature behavior of the system dynamics near the Curie points originates from the fact that the simple Mitsui model does not distinguish between the free and the clamped permittivities. In fact, the relaxation times and dynamic permittivity at frequencies above the frequency of piezoelectric resonance calculated within its framework correspond to a free crystal, which is not correct for Rochelle salt. Instead, like the clamped static dielectric permittivity, the relaxation times calculated for a clamped crystal should be finite at the Curie points. Also, the crystal piezoelectricity contributes to its spontaneous polarization; hence, when this is taken into account, an agreement with the experiment should be improved as well.

The aim of this paper is, by comparing the physical characteristics calculated within the same model for piezoelectric Rochelle salt and non-piezoelectric $\mathrm{RbHSO}_{4}$, to illustrate the role played by piezoelectric interactions in the dielectric response of Rochelle salt and to show that the modified Mitsui model provides a satisfactory and qualitatively correct description of its dielectric characteristics, including the vicinity of the Curie points.

\section{The model}

We start from the conventional Mitsui model and modify it by taking into account the shear strain $\varepsilon_{4}$, spontaneous in the ferroelectric phase or induced by piezoelectric coupling with an external electric field $E_{1}$ applied along the ferroelectric axis $a$. The model Hamiltonian then reads [13]

$$
\begin{aligned}
H= & \frac{v N}{2} c_{44}^{0 E} \varepsilon_{4}^{2}-v N e_{14}^{0} \varepsilon_{4} E_{1}-\frac{v N}{2} \chi_{11}^{0 \varepsilon} E_{1}^{2}-\frac{1}{2} \sum_{q q^{\prime}} \sum_{f f^{\prime}} R_{q q^{\prime}}\left(f f^{\prime}\right) \frac{\sigma_{q f}}{2} \frac{\sigma_{q f^{\prime}}}{2} \\
& -\Delta \sum_{q f}\left(\frac{\sigma_{q 1}}{2}-\frac{\sigma_{q 2}}{2}\right)-\left(\mu_{1} E_{1}-2 \psi_{4} \varepsilon_{4}\right) \sum_{q f} \frac{\sigma_{q f}}{2} .
\end{aligned}
$$

The three first terms in the Hamiltonian represent phenomenological elastic, piezoelectric, and electric energies that do not depend on orientation of quasispins - a lattice contribution; $v$ is the unit cell volume. In the fourth term $R_{q q^{\prime}}\left(f f^{\prime}\right)$ are the potentials of interaction between quasispins. The quantity $\Delta$ describes an asymmetry of the double-well potential; $\mu_{1}$ is the effective dipole moment. The last term in 
the Hamiltonian is an additional internal field produced by piezoelectric coupling with the shear strain $\varepsilon_{4} ; \psi_{4}$ is the so-called deformational potential.

In the case of non-piezoelectric $\mathrm{RbHSO}_{4}$ it suffices to consider the conventional model without strain-related terms

$$
\begin{aligned}
H= & -\frac{v N}{2} \chi_{33}^{0} E_{3}^{2}-\frac{1}{2} \sum_{q q^{\prime}} \sum_{f f^{\prime}} R_{q q^{\prime}}\left(f f^{\prime}\right) \frac{\sigma_{q f}}{2} \frac{\sigma_{q f^{\prime}}}{2} \\
& -\Delta \sum_{q f}\left(\frac{\sigma_{q 1}}{2}-\frac{\sigma_{q 2}}{2}\right)-\mu_{3} E_{3} \sum_{q f} \frac{\sigma_{q f}}{2} .
\end{aligned}
$$

Hereafter we restrict ourselves by the mean field approximation. The obtained thermodynamic potential $g_{1 E}\left(\sigma_{4}, T, E_{1}\right)$ of Rochelle salt ( $\sigma_{4}$ is shear stress conjugate to the strain $\varepsilon_{4}$; in numerical calculations $\left.\sigma_{4}=0\right)$ is [13]

$$
\begin{aligned}
\frac{g_{1 E}}{N k_{\mathrm{B}}}= & -\bar{v} \sigma_{4} \varepsilon_{4}+\frac{\bar{v}}{2} c_{44}^{0 E} \varepsilon_{4}^{2}-\bar{v} e_{14}^{0} \varepsilon_{4} E_{1}-\frac{\bar{v}}{2} \chi_{11}^{0 \varepsilon} E_{1}^{2}-2 T \ln 2+\frac{1}{4}(\tilde{J}+\tilde{K}) \xi^{2} \\
& +\frac{1}{4}(\tilde{J}-\tilde{K}) \sigma^{2}-T \ln \cosh \frac{1}{2}(\gamma+\delta)-T \ln \cosh \frac{1}{2}(\gamma-\delta) .
\end{aligned}
$$

Here $\tilde{J}=\sum_{q} J_{q q^{\prime}} / k_{\mathrm{B}}$ and $\tilde{K}=\sum_{q} K_{q q^{\prime}} / k_{\mathrm{B}}$ are the Fourier transforms of the interaction constants in the same and in different sublattices at $\mathbf{q}=0$. $\xi$ and $\sigma$ are ferroelectric and antiferroelectric order parameters, and

$$
\gamma=\frac{1}{T}\left(\frac{\tilde{J}+\tilde{K}}{2} \xi-2 \tilde{\psi}_{4} \varepsilon_{4}+\mu_{1} E_{1}\right), \quad \delta=\frac{1}{T}\left(\frac{\tilde{J}-\tilde{K}}{2} \sigma+\tilde{\Delta}\right), \quad \bar{v}=\frac{v}{k_{\mathrm{B}}}
$$

for Rochelle salt. For RbHSO 4 :

$$
\begin{aligned}
\frac{g_{1 E}}{N k_{\mathrm{B}}}= & -\frac{\bar{v}}{2} \chi_{33}^{0} E_{3}^{2}-2 T \ln 2+\frac{\tilde{J}+\tilde{K}}{4} \xi^{2} \\
& +\frac{\tilde{J}-\tilde{K}}{4} \sigma^{2}-T \ln \cosh \frac{\gamma+\delta}{2}-T \ln \cosh \frac{\gamma-\delta}{2}
\end{aligned}
$$

and

$$
\gamma=\frac{1}{T}\left(\frac{\tilde{J}+\tilde{K}}{2} \xi+\mu_{3} E_{3}\right)
$$

Spontaneous polarization of Rochelle salt is then given by the expression

$$
P_{1}=e_{14}^{0} \varepsilon_{4}+\chi_{11}^{0 \varepsilon} E_{1}+\frac{\mu_{1}}{v} \xi
$$

whereas for non-piezoelectric $\mathrm{RbHSO}_{4}$ it is

$$
P_{3}=\chi_{33}^{0} E_{3}+\frac{\mu_{3}}{v} \xi .
$$


Now we can easily derive two static dielectric susceptibilities of piezoelectric Rochelle salt: for clamped and free crystals:

$$
\begin{aligned}
& \chi_{11}^{\varepsilon}=\left(\frac{\partial P_{1}}{\partial E_{1}}\right)_{\varepsilon_{4}}=\chi_{11}^{0}+\frac{\beta \mu_{1}^{2}}{2 v} f_{1}(\xi, \sigma), \\
& \chi_{11}^{\sigma}=\left(\frac{\partial P_{1}}{\partial E_{1}}\right)_{\sigma_{4}}=\chi_{11}^{\sigma 0}+\frac{\beta\left(\mu_{1}^{\prime}\right)^{2}}{2 v} f_{2}(\xi, \sigma),
\end{aligned}
$$

where

$$
\begin{aligned}
& f_{1}(\xi, \sigma)=\frac{\rho+\frac{\tilde{K}-\tilde{J}}{4 T}\left[\rho^{2}-4 \xi^{2} \sigma^{2}\right]}{\left[1-\frac{\tilde{K}+\tilde{J}}{4 T} \rho\right]\left[1+\frac{\tilde{K}-\tilde{J}}{4 T} \rho\right]+\frac{\tilde{K}^{2}-\tilde{J}^{2}}{T^{2}} \xi^{2} \sigma^{2}}, \\
& f_{2}(\xi, \sigma)=\frac{\rho+\frac{\tilde{K}-\tilde{J}}{4 T}\left[\rho^{2}-4 \xi^{2} \sigma^{2}\right]}{[1-\rho \zeta]\left[1+\frac{\tilde{K}-\tilde{J}}{4 T} \rho\right]+\zeta \frac{\tilde{K}-\tilde{J}}{T} \xi^{2} \sigma^{2}} \\
& \rho=1-\xi^{2}-\sigma^{2}, \quad \zeta=\frac{\tilde{K}+\tilde{J}}{4 T}+\frac{2}{\bar{v}} \frac{\tilde{\psi}_{4}^{2}}{T c_{44}^{0 E}}, \\
& d_{14}^{0}=\frac{e_{14}^{0}}{c_{44}^{0 E}}, \quad \chi_{11}^{\sigma 0}=\chi_{11}^{0 \varepsilon}+e_{14}^{0} d_{14}^{0}, \quad \mu_{1}^{\prime}=-2 \tilde{\psi}_{4} d_{14}^{0}+\mu_{1} .
\end{aligned}
$$

For non-piezoelectric $\mathrm{RbHSO}_{4}$ there is a single static dielectric permittivity along the axis of spontaneous polarization

$$
\chi_{33}=\chi_{33}^{0}+\beta \frac{\mu_{3}^{2}}{2 v} f_{1}(\xi, \sigma) .
$$

The difference between free and clamped susceptibilities of Rochelle salt

$$
\chi_{11}^{\sigma}-\chi_{11}^{\varepsilon}=e_{14} d_{14}
$$

is given by its coefficient of piezoelectric stress

$$
e_{14}=\left(\frac{\partial P_{1}}{\partial \varepsilon_{4}}\right)_{E_{1}}=e_{14}^{0}-\beta \psi_{4} \frac{\mu_{1}}{v} f_{1}(\xi, \sigma),
$$

and coefficient of piezoelectric strain

$$
d_{14}=\left(\frac{\partial P_{1}}{\partial \sigma_{4}}\right)_{E_{1}}=d_{14}^{0}-\beta \frac{\mu_{1}^{\prime}}{v} \frac{\psi_{4}}{c_{44}^{0 E}} f_{2}(\xi, \sigma) .
$$

Dynamic properties of the system are studied within the Glauber method [14]. Assuming that the strain is time independent (due to clamping of the crystal above the frequency of piezoelectric resonance), at small deviations of the system from equilibrium, we obtain a complex dielectric permittivity with two relaxation times that correspond, in fact, to a clamped crystal [13]

$$
\varepsilon_{11}^{\prime}(\omega)=\varepsilon_{\infty}+\frac{4 \pi \chi_{1}}{1+\left(\omega \tau_{1}\right)^{2}}+\frac{4 \pi \chi_{2}}{1+\left(\omega \tau_{2}\right)^{2}}, \quad \varepsilon_{11}^{\prime \prime}(\omega)=\frac{4 \pi \chi_{1} \omega \tau_{1}}{1+\left(\omega \tau_{1}\right)^{2}}+\frac{4 \pi \chi_{2} \omega \tau_{2}}{1+\left(\omega \tau_{2}\right)^{2}}
$$


where

$$
\begin{aligned}
& \chi_{1,2}=\frac{\mu_{1}^{2}}{v k_{B}} \frac{1}{2 T} \frac{\tau_{1} \tau_{2}}{\tau_{2}-\tau_{1}}\left(\mp K^{(1)} \pm \tau_{1} K^{(0)}\right), \quad \tau_{1,2}^{-1}=\frac{1}{2}\left\{K_{1} \mp \sqrt{K_{1}^{2}-4 K_{0}}\right\}, \\
& K^{(1)}=-a_{1}, \quad K_{1}=a_{11}+a_{22}, \quad K^{(0)}=-\left|\begin{array}{ll}
a_{12} & a_{1} \\
a_{22} & a_{2}
\end{array}\right|, \quad K_{0}=\left|\begin{array}{ll}
a_{11} & a_{12} \\
a_{21} & a_{22}
\end{array}\right| .
\end{aligned}
$$

Also,

$$
\begin{array}{rlrl}
a_{11} & =1-\frac{\tilde{J}+\tilde{K}}{T} \frac{1-\cosh \gamma \cosh \delta}{(\cosh \gamma+\cosh \delta)^{2}}, & & a_{12}=\frac{\tilde{J}-\tilde{K}}{T} \frac{\sinh \gamma \sinh \delta}{(\cosh \gamma+\cosh \delta)^{2}}, \\
a_{21}=\frac{\tilde{J}+\tilde{K}}{T} \frac{\sinh \gamma \sinh \delta}{(\cosh \gamma+\cosh \delta)^{2}}, & a_{22}=1-\frac{\tilde{J}-\tilde{K}}{T} \frac{1-\cosh \gamma \cosh \delta}{(\cosh \gamma+\cosh \delta)^{2}} \\
a_{1}=\frac{4(1-\cosh \gamma \cosh \delta)}{(\cosh \gamma+\cosh \delta)^{2}}, & a_{2}=\frac{4 \sinh \gamma \sinh \delta}{(\cosh \gamma+\cosh \delta)^{2}} .
\end{array}
$$

The corresponding expressions for the dynamic permittivity of $\mathrm{RbHSO}_{4}$ are obtained by taking the appropriate expression for $\gamma(5)$ and changing $\mu_{1} \rightarrow \mu_{3}$.

It can be shown that one of the relaxation times $\left(\tau_{1}\right)$ exhibits a critical slowing down at the transition points, whereas the other one has only bends at these temperatures. Since the weight $\chi_{1}$ corresponding to $\tau_{1}$ is several orders larger than $\chi_{2}$, and, furthermore, $\chi_{2}$ is different from zero only in the ferroelectric phase, the dielectric relaxation in these crystals is of Debye type.

\section{Discussion}

Values of the theory parameters providing the best possible description of experimental data for Rochelle salt and $\mathrm{RbHSO}_{4}$ are presented in table 1. Details of the fitting procedure are given elsewhere $[2,13]$. After fulfilling the foremost task to reproduce the observable number of the transition points in the crystals and their temperatures, for $\mathrm{RbHSO}_{4}$ the next main criterion for choosing values of the theory parameters was to obtain the best fit to the not too large but perceptible jump of specific heat at the transition point [15]. On the contrary, since no reliable experimental data for the peculiarities of the specific heat of Rochelle salt are available (even the signs of its anomalies at the Curie points have not been firmly established; see [16]), for this crystal we chose those values of the model parameters, which provide the best fit to spontaneous polarization, piezoelectric module $d_{14}$, and elastic constants $c_{44}^{\mathrm{E}}$ and $c_{44}^{\mathrm{P}}$. For comparison we also present here the results obtained for Rochelle salt within a conventional Mitsui model without piezoeffect. In this case the values of the free parameters were chosen [2] such as the best description of static dielectric susceptibility is obtained.

Figure 1 shows that the theory without piezoelectric coupling provides a satisfactory agreement with the experiment both for static susceptibility near the Curie point and for spontaneous polarization of $\mathrm{RbHSO}_{4}$. As seen in figure 2, the theory 
Table 1. Values of the model parameters for Rochelle salt and $\mathrm{RbHSO}_{4}$. For Rochelle salt also $c_{44}^{E 0}=12.8 \cdot 10^{10} \mathrm{dyn} / \mathrm{cm}^{2}, d_{14}^{0}=1.9 \cdot 10^{-8} \mathrm{esu} / \mathrm{dyn}$.

\begin{tabular}{|c|c|c|c|c|c|c|c|}
\hline & $J$ & $K_{(\mathrm{K})}$ & $\Delta$ & $\psi_{4}$ & $\begin{array}{c}\alpha \\
\left(10^{-13} \mathrm{c}^{-1}\right)\end{array}$ & $\underset{\left(10^{-18} \stackrel{\mu}{\mu} \mathrm{esu} \cdot \mathrm{cm}\right)}{ }$ & $\begin{array}{c}v \\
\left(10^{-21} \mathrm{~cm}^{3}\right)\end{array}$ \\
\hline Rs & 797.36 & 1468.83 & 737.33 & -760 & 1.7 & $2.52+0.0066(297-T)$ & 0.5219 \\
\hline Rs & 802.12 & 1473.59 & 737.3 & - & 0.9 & 1.84 & 0.5219 \\
\hline $\mathrm{RbHSO}_{4}$ & 780.9 & 880.64 & 398.8 & - & 0.48 & 0.425 & 0.2105 \\
\hline
\end{tabular}

without piezoeffect also yields a good description of the temperature and frequency dependences of dynamic dielectric permittivity of $\mathrm{RbHSO}_{4}$. Note that due to divergence of the relaxation time $\tau_{1}$, both experimental and theoretical curves of the real part of permittivity turn to $\varepsilon_{\infty}$ at the transition point.
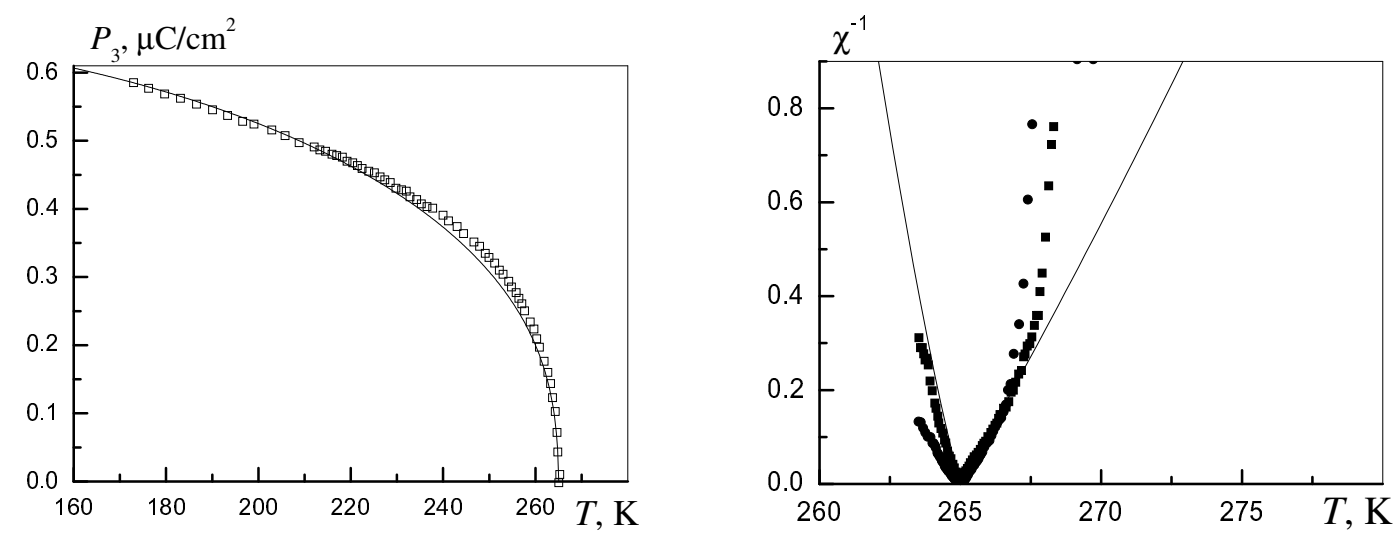

Figure 1. Temperature dependence of polarization and static dielectric susceptibility of $\mathrm{RbHSO}_{4}$. Experimental points taken from [17].

In figure 3 we plot the temperature dependences of inverse static dielectric susceptibilities of Rochelle salt. One can see a clear difference between free and clamped susceptibilities. At the transition points, the static dielectric susceptibility of a free crystal diverges, whereas the susceptibility of a clamped crystal remains finite. The chosen value of $\mu_{1}$ provides a satisfactory description of experimental data for the free susceptibility in the ferroelectric and high-temperature paraelectric phases.

Theoretical dependence of spontaneous polarization $P_{1}$ of Rochelle salt is depicted in figure 4. Due to the contribution of piezoelectric coupling, a description of experimental data, though still not satisfactory, is much better than within the theories that do not take into account the piezoelectric effect. The calculated maximal value of $P_{1}$ is lower here than the experimental one only by $10 \%$, as compared to nearly $50 \%$, obtained within the conventional model.

Figure 5 shows that a conventional theory without piezoelectric coupling fails to describe a temperature behavior of relaxation time $\tau_{1}$ (the one exhibiting critical slowing down) and real part of dynamic dielectric permittivity near the Curie 

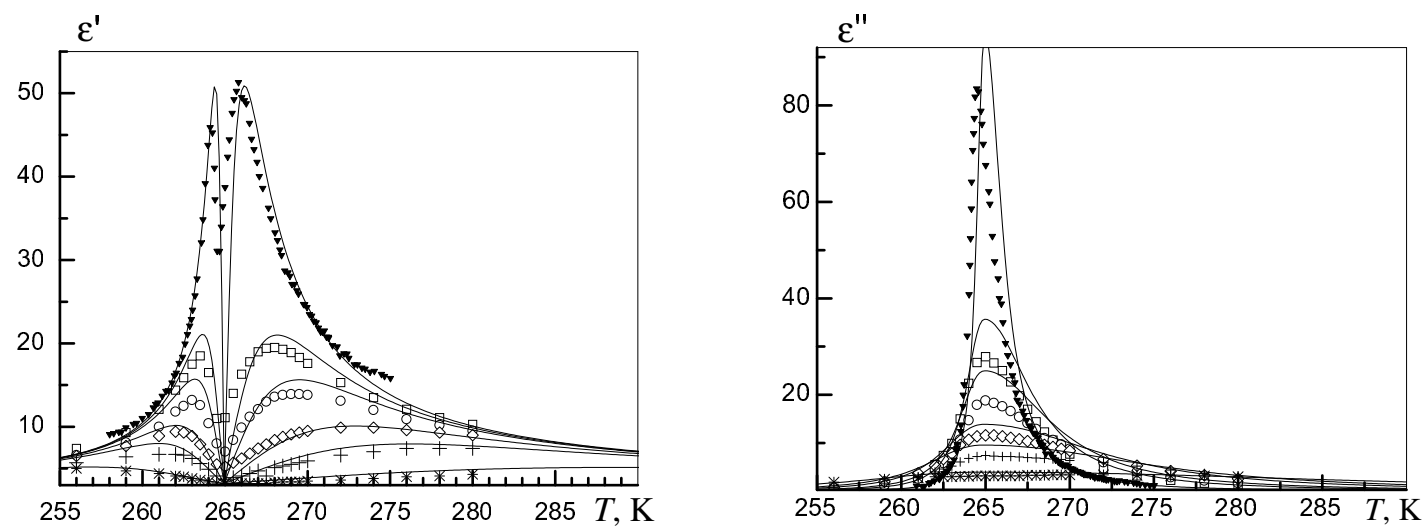

Figure 2. Temperature dependences of real and imaginary parts of dynamic dielectric permittivity of $\mathrm{RbHSO}_{4}$ at different frequencies $(\mathrm{GHz}): \mathbf{\nabla}-3.27, \square-$ $8.75, \circ-12.5, \diamond-22.5,+-32.6, \times-78.8$. Experimental points are taken from $[10]$.

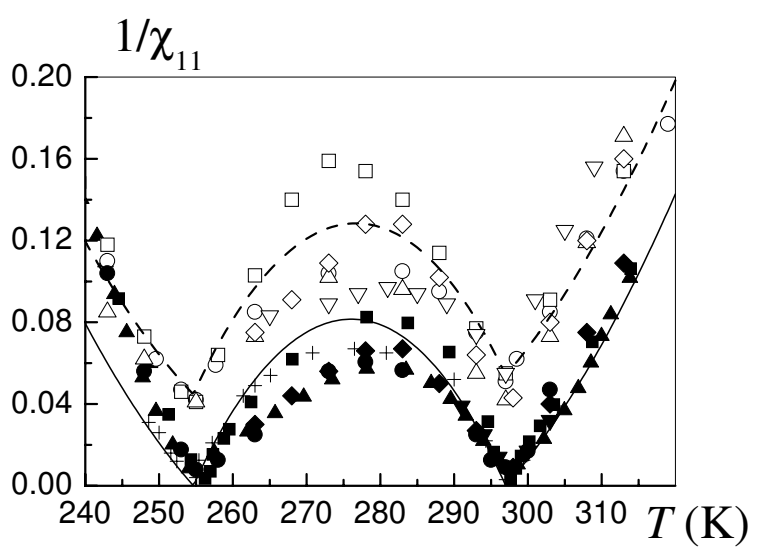

Figure 3. Temperature dependence of inverse static dielectric susceptibility of a free: $\mathbf{\square}-[18], \boldsymbol{\Delta}-[19], \boldsymbol{-}-[20]$, $[21], \quad \boldsymbol{\nabla}-[22], \quad+-[23]$ and clamped: $\square-[9], \quad O_{-}[24], \diamond-[20], \triangle-[25]$, $\nabla-[26]$ crystals of Rochelle salt.

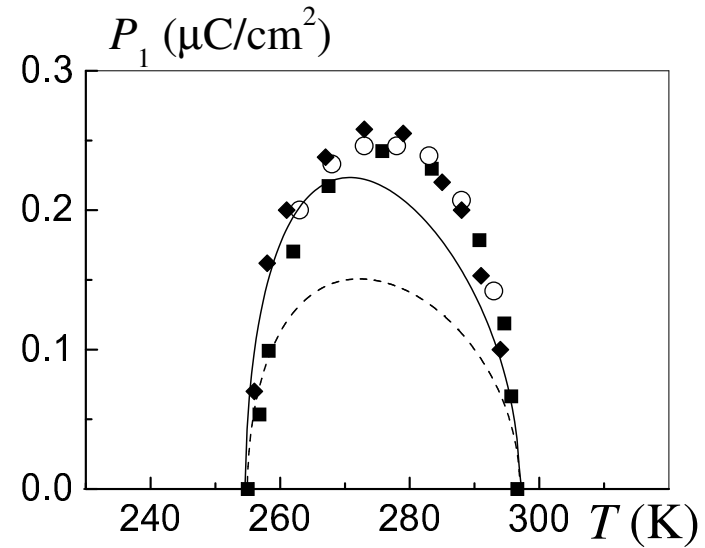

Figure 4. Spontaneous polarization of Rochelle salt. Solid and dashed lines are obtained within the theories with piezoeffect and without it. Experimental points are: $\mathrm{O}_{-}[27], \mathbf{\square}-[18]$, [20]. 

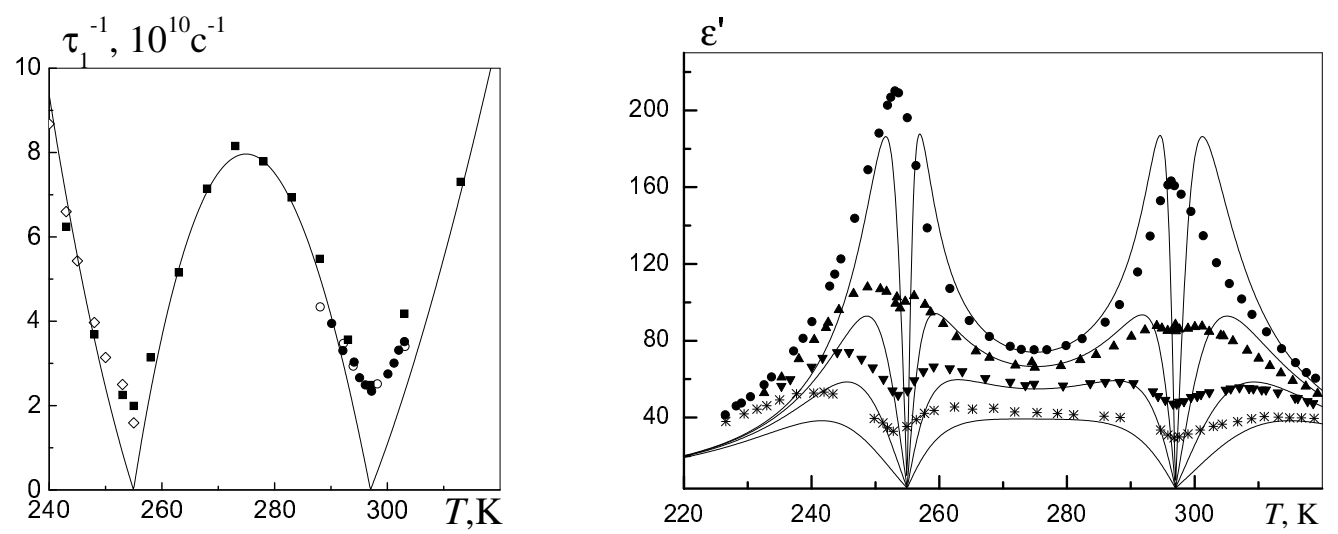

Figure 5. Inverse relaxation time (left) and real part of the dynamic dielectric permittivity of Rochelle salt at different frequencies $(\mathrm{GHz}): \bullet-2.5, \mathbf{\Delta}-5.1, \boldsymbol{\nabla}-$ $8.25, *-12.95$ (right) calculated within the theory without piezoelectric effect. Experimental points for relaxation time are taken from $\bullet-[28], \quad O_{-}[29]$, $[9], \diamond-[30]$ and for permittivity they are taken from [9].
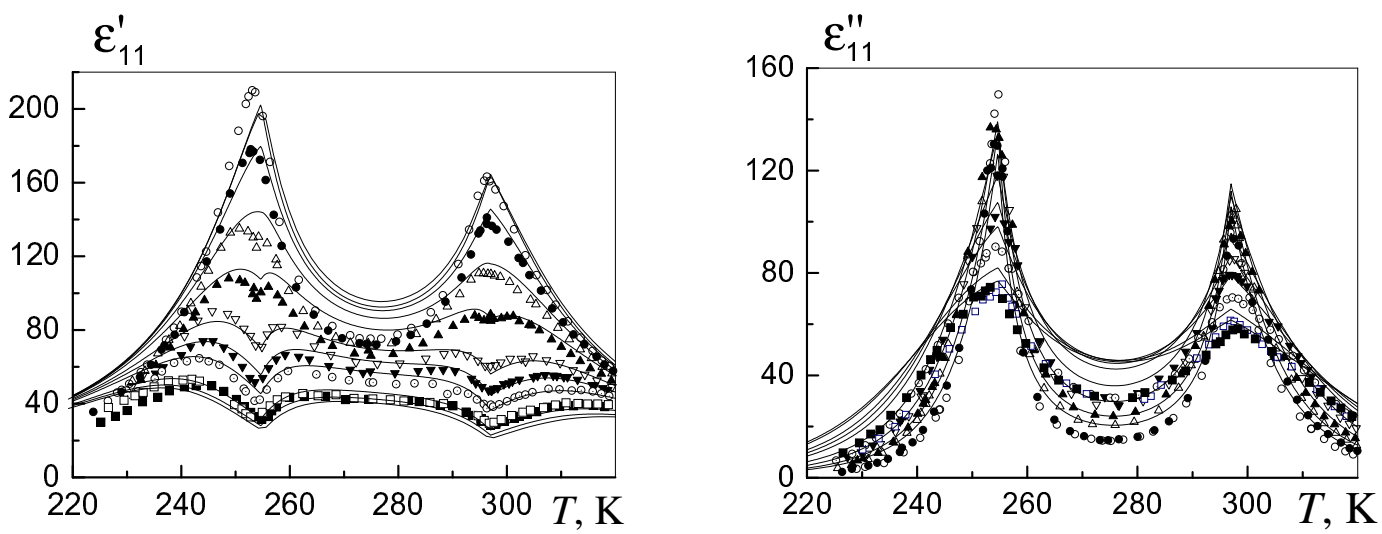

Figure 6. Real and imaginary parts of the dynamic dielectric permittivity of Rochelle salt at different frequencies $(\mathrm{GHz}): \mathrm{O}_{-}-2.5, \bullet-3, \triangle-3.9, \boldsymbol{\Delta}-5.1$, $\nabla-7.05, \boldsymbol{\nabla}-8.25, \odot-9.45, \square-11.96, \mathbf{\square}-12.95$. Experimental points are taken from $[9]$.

points. As we have mentioned earlier, this theory does not distinguish between permittivities of free and clamped crystals and yields a diverging relaxation time $\tau_{1}$ and, therefore, causes a vanishing contribution of the ordering subsystem to the real part of permittivity.

The modified model is free from this drawback, since it permits to calculate the clamped relaxation time and permittivity. The relaxation time, as well as the static dielectric susceptibility $\varepsilon_{11}^{\varepsilon}$, would hypothetically diverge at two temperatures within the ferroelectric phase, a few degrees away from the Curie points. In reality, both $\tau_{1}$ and $\varepsilon_{11}^{\varepsilon}$ always stay finite and, thus, the contribution of the ordering subsystem to the dynamic permittivity always remains different from zero as well. The piezoelectric effect being taken into account yields a correct temperature behavior of inverse relaxation times, which now have two finite minima at the Curie points. 
Figure 6 illustrates the fact that under a proper choice of the parameter $\alpha$ that sets a time scale of the dynamic processes in the system, we obtain a qualitatively correct and quantitatively fair description of experimental data for dynamic permittivity of Rochelle salt, including the vicinity of the Curie points.

\section{Acknowledgements}

We thank Prof. M.O.Romanyuk as well as participants of the VI UkrainianPolish and II East-European Meeting on Ferroelectrics Physics (Synjak, Ukraine, September 6-10, 2002) for valuable and helpful discussions.

\section{References}

1. Mitsui T. // Phys. Rev., 1958, vol. 111, p. 1259.

2. Levitskii R.R., Verkholyak T.M., Kutny I.V., Hil I.G. Investigation of ferroelectric order-disorder type compounds with asymmetric double-well potential. Preprint condmat/0106351.

3. Kalenik J. // Acta Phys. Pol. A, 1975, vol. 48 p. 387.

4. Zeks B., Shukla G.C., Blinc R. // Phys. Rev. B., 1971, vol. 3, p. 2306.

5. Zeks B., Shukla G.C., Blinc R. // J. Phys., 1972, vol. 33, supplement to No. 4, c2-67c2-68.

6. Levitskii R.R., Zachek I.R., Varanitskii V.I. // Ukrainian Journal of Physics, 1980, vol. 25, p. 1766 (in Russian).

7. Levitskii R.R., Antonyak Yu.T., Zachek I.R. // Ukrainian Journal of Physics, 1981, vol. 26, p. 1835 (in Russian).

8. Levitskii R.R., Sokolovskii R.O. // Condens. Matter Phys., 1999, vol. 2, p. 393.

9. Sandy F., Jones R.V. // Phys. Rev., 1968, vol. 168, p. 481.

10. Grigas J., Zachek I.R., Krasikov V.S., Kutny I.V. et al. // Lithuanian Physics Proceedings, 1984, vol. 24, p. 33 (in Russian).

11. Levitskii R.R., Zachek I.R., Varanitskii V.I. // Fiz. Tverd. Tela (Leningrad), 1980, vol. 22 , p. 2750.

12. Ambrazyavichene V.A., Volkov A.A., Kozlov G.V., Krasikov V.S., Kryukova E.B. // Fiz. Tverd. Tela (Leningrad), 1983, vol. 25, p. 1605.

13. Levitskii R.R., Zachek I.R., Verkholyak T.M., Moina A.P. // Phys. Rev. B, 2003, vol. 67 , No. 17 , p. 174112.

14. Glauber R.J. // J. Math. Phys., 1963, vol. 4, p. 294.

15. Alexandrov K.S., Anistratov A.T. // Ferroelectrics, 1976, vol. 12, p. 191-193.

16. Dey P.K., Som K.K., Chowdhury K.R., Sarkar B.K., Chaudhuri B.K. // Phys. Rev. B, 1993, vol. 47, p. 3001.

17. Kajikawa H., Ozaki T., Nakamura E. // J. Phys. Soc. Jpn., 1977, vol. 43, p. 937-941.

18. Hablützel J. // Helv. Phys. Acta, 1939, vol. 12, p. 489.

19. Taylor W., Lockwood D.J., Labbe H.J. // J. Phys. C.: Solid State Phys., 1984, vol. 17, p. 3685-3699.

20. Mueller H. // Phys. Rev., 1935, vol. 47, p. 175.

21. Mason W.P. // Phys. Rev., 1939, vol. 55, p. 775.

22. Petrov V.M. // Kristallogr., 1962, vol. 7, p. 403-407 (in Russian). 
23. Yurin V.A. // Bull. Ac. Sci. USSR, 1965, vol. 29, p. 2001-2004 (in Russian).

24. Mason W.P. // Phys. Rev., 1947, vol. 72, p. 854.

25. Akao H., Sasaki T. // J. Chem. Phys., 1955, vol. 23, p. 2210.

26. Gutin L. // Journ. Exp. Theor. Phys., 1945, vol. 15, p. 199-207 (in Russian).

27. Cady W.G. Piezoelectricity: an Introduction to the Theory and Application of Electromechanical Phenomena in Crystals. New York, London McGraw-Hill, 1946.

28. Müser H.E., Pottharst J. // Phys. Stat. Sol., 1967, vol. 109.

29. Kolodziej H. - In: Dielectric and Related Molecular Processes. Vol. 2, London WIVOBN, The Chemical Society Burlington House, 1975, p. 249-287.

30. Volkov A.A., Kozlov G.V., Lebedev S.P. // Zh. Eksp. Teor. Fiz., 1980, vol. 79, p. 1430.

\title{
Роль п'єзоелектричного ефекту у діелектричному відгуку кристалів типу сегнетової солі
}

\author{
Р.Р.Левицький ${ }^{1}$, І.Р.Зачек ${ }^{2}$, Т.М.Верхоляк ${ }^{1}$, А.П.Моїна ${ }^{1}$ \\ 1 Інститут фізики конденсованих систем НАН України, \\ 79011 Львів, вул. Свєнціцького, 1 \\ 2 Національний університет "Львівська політехніка", \\ 79013 Львів, вул. Бандери, 12
}

Отримано 9 грудня 2002 р.

Шляхом порівняння поведінки спонтанної поляризації та статичних і динамічних діелектричних характеристик п'єзоактивного кристалу сегнетової солі та неп'єзоелектричного $\mathrm{RbHSO}_{4}$ вивчається роль п'єзоелектричної взаємодії у діелектричному відгуку кристалів цього типу. Розрахунки для обидвох кристалів проводяться в рамках моделі Міцуї, модифікованої для випадку сегнетової солі шляхом врахування доданків, пов'язаних з п'єзоелектричною взаємодією зі спонтанною деформацією $\varepsilon_{4}$. Показано, що така модифікація моделі покращує узгодження між теорією та експериментом для спонтанної поляризації та забезпечує правильну температурну поведінку часів релаксації та динамічної діелектричної проникності сегнетової солі в околі точок переходу.

Ключові слова: сегнетова сіль, $\mathrm{RbHSO}_{4}$, п'єзоефект, релаксаційна динаміка, затискання

PACS: 77.22.Gm, 77.65.-j, 77.80.Bh 